\title{
High temperature piezoelectric drill
}

\author{
Xiaoqi Bao ${ }^{1}$, Yoseph Bar-Cohen ${ }^{1}$, Stewart Sherrit ${ }^{1}$ and Mircea Badescu ${ }^{1}$ and Tom Shrout ${ }^{2}$ \\ 1 Jet Propulsion Laboratory, California Institute of Technology, Pasadena, CA 91109-8099, \\ xbao@jpl.nasa.gov \\ 2 Pennsylvania State University, University Park, PA 16802
}

\begin{abstract}
Venus is one of the planets in the solar systems that are considered for potential future exploration missions. It has extreme environment where the average temperature is $460^{\circ} \mathrm{C}$ and its ambient pressure is about $90 \mathrm{~atm}$. Since the existing actuation technology cannot maintain functionality under the harsh conditions of Venus, it is a challenge to perform sampling and other tasks that require the use of moving parts. Specifically, the currently available electromagnetic actuators are limited in their ability to produce sufficiently high stroke, torque, or force. In contrast, advances in developing electro-mechanical materials (such as piezoelectric and electrostrictive) have enabled potential actuation capabilities that can be used to support such missions. Taking advantage of these materials, we developed a piezoelectric actuated drill that operates at the temperature range up to $500^{\circ} \mathrm{C}$ and the mechanism is based on the Ultrasonic/Sonic Drill/Corer (USDC) configuration. The detailed results of our study are presented in this paper.
\end{abstract}

KEYWORD: High Temperature, Piezoelectric Drill, Rock sampling, Venus.

\subsection{INTRODUCTION}

Future NASA missions will require a variety of new technologies in order to enable operation at planets with extreme conditions in the Solar System [Gershman and Wallace, 1999]. Venus is one of the planets that are targeted for a mission in the current NASA Decadal study of potential new Frontier missions and it is considered a planet with a hostile environment where the average temperature is $460^{\circ} \mathrm{C}$ and its ambient pressure is about $90 \mathrm{~atm}$. Since the existing actuation technology cannot maintain functionality under the harsh conditions of Venus, it is a challenge to perform sampling and other tasks that require the use of moving parts. Specifically, the currently available electromagnetic actuators are limited in their ability to produce sufficiently high stroke, torque, or force. In contrast, advances in developing electro-mechanical materials (such as piezoelectric and electrostrictive) have enabled potential actuation capabilities that can be used to support such missions. Prior to this task, we studied several promising piezoelectric ceramics and single crystal materials to determine the feasibility of using these materials as actuators. We identified several piezoelectric materials that have the potential to provide the required characteristics [Sherrit et al., 2004] and were used in this report study.

Many issues have to be considered when developing devices for operation at high temperature. These issues include material compatibility, chemical reactions, alloying, annealing and diffusion characteristics that can affect the chemical and physical nature of components that are being used. Thermal expansion mismatch can be catastrophic to the components that are required to fit precisely inside a structure. Further, the required piezoelectric materials have to operate effectively and temperatures around $500^{\circ} \mathrm{C}$ are outside the normal operating range for standard piezoelectric, ferroelectric and ferromagnetic material based actuators due to the transition temperature (i.e., Curie temperature) where these materials switch from Ferro to Para causing them to lose their actuation capability. Such actuators as brush, brushless, and stepper motors require magnetic materials where some of the commercial units can be operated at approximately $200-300^{\circ} \mathrm{C}$, although recently developed motor at Honeybee [Bar-Cohen and Zacny, 2009] and JPL [Troy 2011] were reported to operate at much higher temperatures. In these actuators the primary breakdown mechanism is shorting in the windings insulation rather than operation above or near the Curie temperature. Some motors designed for extraction of smoke and deadly toxic fumes during fire emergencies are available for operation at the required range. However, they are usually large and have lifetime of a couple of hours.

We investigated the use of HT piezoelectric materials since they can be attached to actuated structures, do not require windings of electric wires, and do not require electrical or mechanical commutation. In parallel to the development of effective actuation materials, we constructed an ultrasonic/sonic driller/corer (USDC) as a sampler using the developed materials. The materials that we investigated in this task include: Lithium Niobate $\left(\mathrm{LiNbO}_{3}\right) \mathrm{Y}$ cut $36^{\circ}$ (has a Curie temperature of $1150^{\circ} \mathrm{C}$ ), as well as Bismuth Titanate materials with various Tungsten doping (produced by our team partners at Penn State University). 


\section{INVESTIGATING MATERIAL PROPERTIES AT HIGH TEMPERATURE}

We evaluated and characterized high temperature piezoelectric material samples towards producing effective HT sampler breadboards. For this purpose, we used an Agilent 4294A Precision Impedance Analyzer and a d33 meter to measure the electrical characteristics at both room and high temperatures up to $500^{\circ} \mathrm{C}$. In parallel to making new HT piezoelectric materials, the electromechanical properties of single crystal Y-cut LiNbO3 discs were investigated to determine the aging characteristics. Isothermal tests at $500^{\circ} \mathrm{C}$ for 1000 hours yielded no appreciable change in properties (to the level of the measurement error) demonstrating its high stability. Also, 3 types of electrodes were tested including 1) sputtered platinum film, 2) gold-palladium thick film and 3) sputtered gold film. The use of sputtered gold as electrodes was found to sustain severe degradation while the Pd-Au and Pt electrodes operated quite effectively. Based on these results and the fabrication ease, sputtered platinum films were chosen as the electrodes.

The first high temperature candidate piezoelectric material that was developed in this study was based on Bismuth Titanate with various composition levels of Titanate and dopants (W, Fe, Ca, Sr, and Mn). The test results have shown significant capability compared to the known materials where the thickness coupling coefficient at $500^{\circ} \mathrm{C}$ is about 15 to $20 \%$. Independent of the first batches that were identified to have a reasonable response the tuning of the dopant additive of Tungsten into the powder mixture were tested. Effective mixtures were identified and HT piezoelectric rings were made using hot isostatic pressing to insure the production of robust low porosity ceramic compositions. Bismuth Titanate 1.0 and 1.5 inch diameter rings, made with effective Titanate concentrations, were produced and were used to fabricate USDC-based samplers. In addition, sampler units were made that were actuated by 1.0 and 2.0 inch diameter LiNbO3 rings.

\section{TESTBED SETUP}

In order to test the developed sampler at as high as $500^{\circ} \mathrm{C}$, a testbed HT chamber was developed and instrumented to monitor the temperature of the drill inside the chamber as well as control the preload and track the drilling rate as a function of various test parameters. The testbed was designed to allow for placing the drill bit of the HT USDC against a rock and controlling the preload of the drill. The testbed consisted of a commercial horizontal tube shape furnace with customized insulation side caps that have a center hole (see on the right in Figure 1). This hole was used in the initial studies for inserting the bit where the drill was kept outside the chamber (Figure 1) and later the drill was inserted completely inside the chamber while the cable was inserted thru the hole. To control the preload and feed rate a pushrod controlled by a pneumatic cylinder was used (behind the USDC in the photo of Figure 1). The preload controller was driven by air from an air cylinder with 3/4" bore diameter (Model Norgren RLC09A), which was mounted on a frame behind the support fixture to push the sampler forward. The pressure in the cylinder and, then, the pushing force was controlled by Omega Electropneumatic Transducer CS-2250. A dial indicator with resolution of 0.001" (0.0254 mm) was used to measure the forward distance of the shaft of the cylinder. In this configuration, limestone was drilled at room temperature (RT) reaching a depth of $22.3 \mathrm{~mm}$ in $23 \mathrm{~min}$, while at $500^{\circ} \mathrm{C}$ a depth of slightly greater than $2 \mathrm{~mm}$ was reached. The bit was made of Crucible hardened CPM-3V with hardness (HRC 59) and degradation of the cutting teeth of the bit was observed at $500^{\circ} \mathrm{C}$ drilling necessitating use of a more durable material.

To test the USDC with the HT piezoelectric stacks inside the chamber we constructed a fixture that is shown schematically and photographically in Figure 2. This fixture allows the sliding of the USDC while drilling as well as securing the position of the drilled rocks along the bit path. The USDC is pushed from outside the chamber using the controlled preload system that developed and tested.
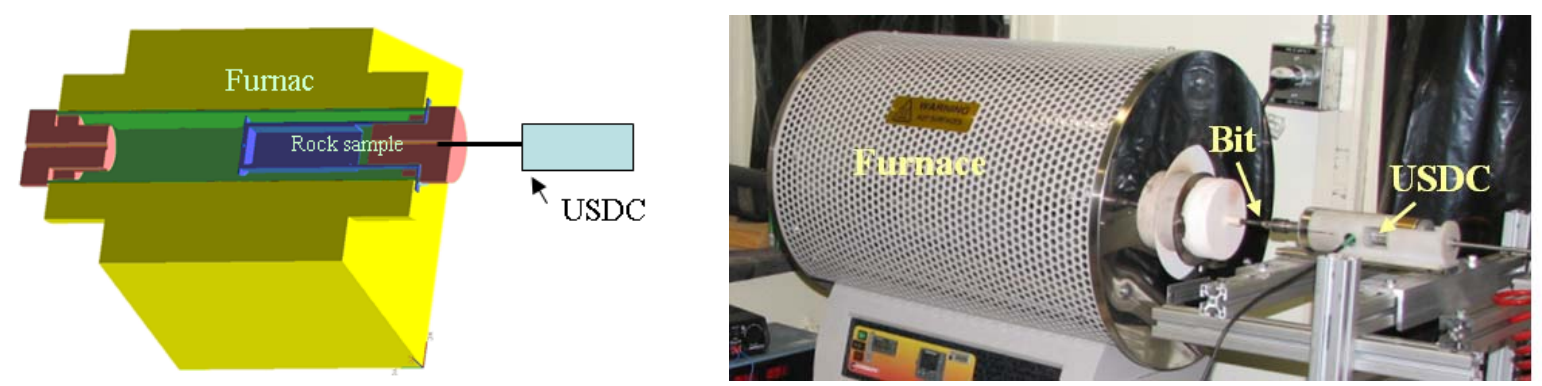

Figure 1: Schematic cross-section and photographic views of the furnace of the developed HT testbed. For the initial testing, rock samples were placed inside the chamber and were drilled at various temperatures while the USDC was placed outside and the preload was controlled pneumatically. 
The driving power was provided by a power amplifier ENI1104LA with an input from Tektronix AFG 3022 Dual Channel Function Generator. The later was programmed to generate sine-wave with controlled duty cycle (e.g., on for 1 second per 2 seconds cycles). A custom made transformer with a voltage ratio factor of up to 1:5 was placed between the amplifier and the sampler to improve the impedance match to the ultrasonic transducer. A 1 Ohm resistor was connected to the sampler input in series as a current-voltage converter for the monitoring oscilloscope. The driving voltage and current were monitored and recorded by a digital oscilloscope (Model Tektronix TDS5054B-NV). The input electric power was calculated in real time by programming the oscilloscope. The instrumented chamber that makes up our testbed is shown photographically in Figure 3.

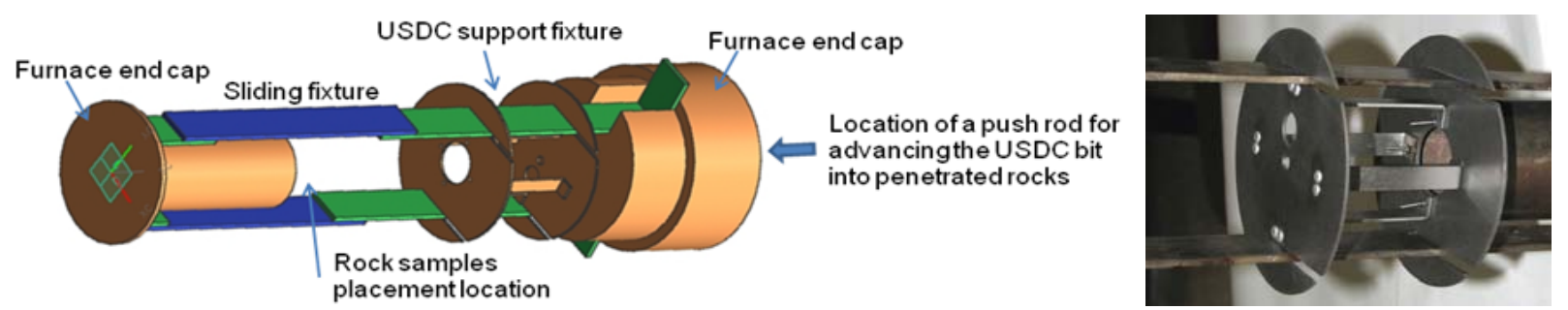

Figure 2: Schematic and photographic views of the support fixture for testing the USDC and its drilling performance inside the HT chamber. The photograph shows a close-up of the USDC mount.

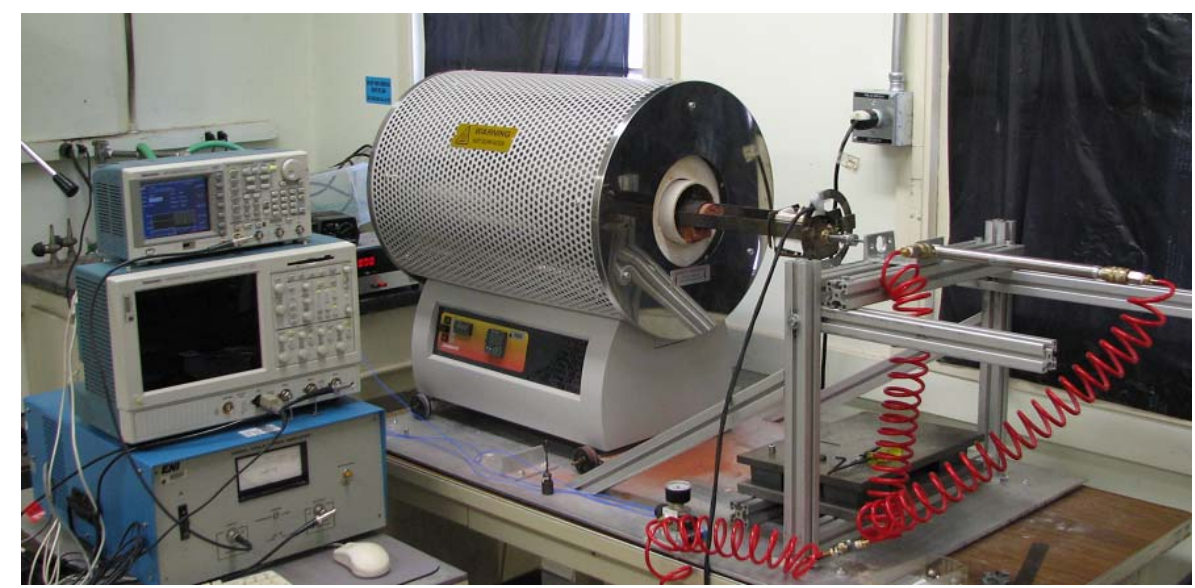

Figure 3: The high temperature testbed setup including the instrumented chamber, the support of the sampler (with the sampler and sample), the pneumatic pushing setup as well as the drive and measurement instruments.

\section{MODELING AND ANALYSIS OF THE TRANSDUCERS}

The transducer that drives the USDC is the key to its operation as a sampler and it is essential to optimize its design to maximize the drilling capability using piezoelectric materials with relatively low performance. For this purpose, finite element models were established to analyze configurations of the transducer that are based on the room temperature baseline USDC transducer design [Bar-Cohen and Zacny, 2009] (see Figure 4). This design consists of a piezoelectric stack, horn, backing block, and pre-stress bolt. Using $\mathrm{LiNbO}_{3}$ single crystal the admittance response was determined for frequencies around the first resonance (Figure 5) suggesting resonance frequency and mode shape similar to that using PZT-8 (Figure 6). However, the maximum conductance is much lower implying that the actuation power under the same voltage will be much lower. Increasing the driving voltage partially compensates for the disadvantage of the high input resistance, which is ultimately limited by electric break down. Further, the high loss at high temperatures may result in quick overheating when operating the drill without duty cycling.

Longer or larger diameter LN transducers are predicted to have improved performances although, at $500^{\circ} \mathrm{C}$, they are still poorer than the reference transducer of PZT- 8 by a factor of 5-6 as judged by the tip velocity for the same driving voltage and Mechanical Q. Increasing the voltage may help compensating for the lower performance. A longer and thicker design was considered to improve the performance further. 


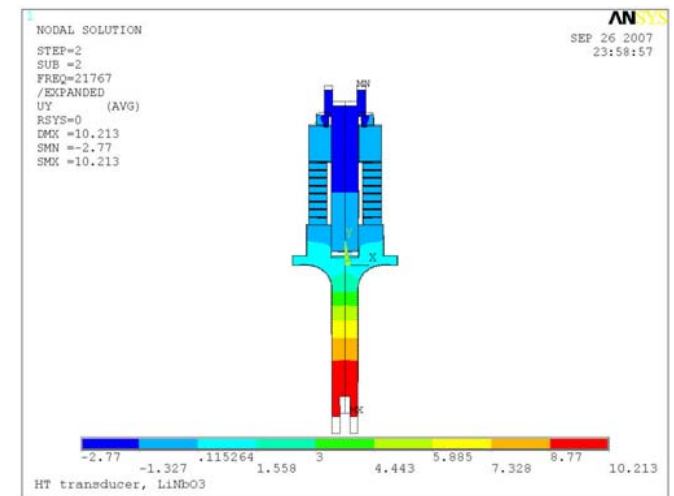

Figure 4: The first longitudinal mode of a LiNbO3 shape with resonance frequency at $21.767 \mathrm{kHz}$. The color scale shows the displacement in the vertical direction.

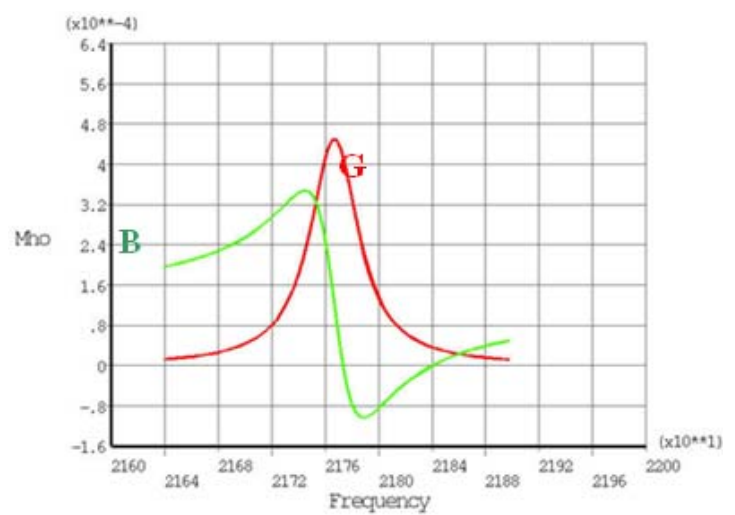

Figure 5: The input admittance response around the first resonance frequency. $\mathrm{G}$ is the real part (conductance) and $\mathrm{B}$ is the imaginary part. The mechanical Q is set as 500 .

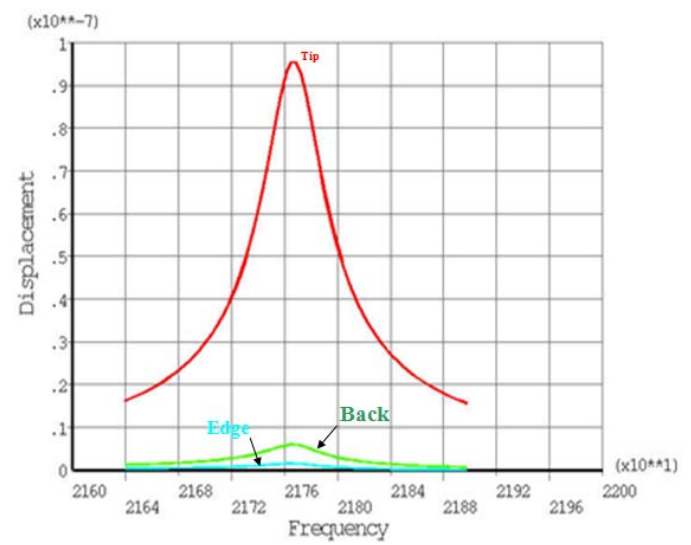

Figure 6: The displacement distribution as a function of frequency for LiNbO3 at the tip of the horn, as well as the back of the transducer as well as the plane of the mounting frame.

\subsection{Impedance Analysis at High Temperature with Low and High Voltage}

To analyze the performances of the breadboard transducer, impedance scans were performed using small signals by an Agilent 4294A precision impedance analyzer with voltage level of $0.5 \mathrm{~V}$. In addition, the measurement was also done using large signals with an in-house setup. A higher voltage at the level close to the operating voltage was applied and the measurements were done at both room temperature and high temperature of $500^{\circ} \mathrm{C}$. A computer program was developed to extract the parameters of equivalent circuit for both small signal and large signal measurements data. Although the Agilent 4294A impedance analyzer has the function to extract the parameters, the error in the extracted values are very large when the electric loss of the piezoelectric elements are relatively high since the equivalent circuit model used by the analyzer neglected the dielectric and piezoelectric loss. The electric loss of these piezoelectric materials at high temperature is quite high, much higher than at room temperature. A model including the electric loss was established and a computer program was developed to extract the parameters from the measured data. The details of the analysis and the results have been reported in [Bao et al 2010].

\section{HT USDC SAMPLER BREADBOARD}

\subsection{Basic configurations}

The components of a USDC that can operate at high temperatures were produced to support the testing of the developed piezoelectric stacks. The key components of the USDC are the actuator, free-mass and bit. A schematic diagram of the USDC is shown in Figure 7. To make sure that the actuator can operate at the required temperature of $500^{\circ} \mathrm{C}$, the horn was fabricated of Titanium and the stress bolt and backing of stainless steel and Inconel Belleville washers were used to increase thermal stability and maintain the piezoelectric stack pre-stress as the actuator is heated. The use of titanium allowed for producing a low mass device that has high mechanical strength. The $\mathrm{LiNbO}_{3}$ or piezoelectric ceramic discs 
were mounted into the actuator and held in compression by the stress bolt to maintain good acoustic coupling between the parts during operation. The design parameters of the actuator were determined by model analysis and the impendence characteristics of the actuator were tested at RT and $500^{\circ} \mathrm{C}$ using the HT chamber. The first HT drill bit was made of Crucible hardened CPM-3V but under the exposure to $500^{\circ} \mathrm{C}$ it was degraded as a result of drilling. As a replacement for the drill bit material, a Tungsten Carbide WA-2 was used and it is considered one of the hardest commercially available materials having hardness of (HRA 92).

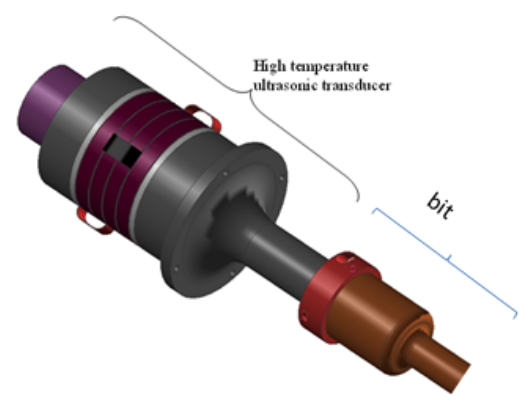

Figure 7: Schematic view of the HT USDC that is being developed.

\section{RESULTS OF BREADBOARD SAMPLER TESTING}

\subsection{Samplers actuated by a 1" diameter HT piezoelectric discs}

Three different piezoelectric materials in a 1" sampler configuration were used to drill brick samples at both room temperature and at $500^{\circ} \mathrm{C}$ mounted inside the chamber. The drilling was stable at room temperature, however, at high temperature the drilling started with a lower rate and dropped quickly especially for the $\mathrm{LiNbO}_{3}$ sampler. A summary of the maximum drilling rates and driving conditions are given in Table 1. The $\mathrm{LiNbO}_{3}$ sampler initial drilling only lasted for 2 minutes

Table 1: Summary of drilling test of 1" samplers

\begin{tabular}{|l|c|c|c|c|c|c|c|c|}
\hline & \multicolumn{4}{|c|}{$23 \mathrm{C}$} & \multicolumn{4}{c|}{$500 \mathrm{C}$} \\
\hline & V rms & A rms & Minutes & Drill Rate (mm/min) & Voltage & A rms & Minutes & Drill rate (mm/min) \\
\hline PZ46 & 1320 & 0.4 & 12 & 1.94 & 830 & 0.37 & 10 & 0.02 \\
\hline PSU & 900 & 0.35 & 7 & 1.1 & 460 & 0.3 & 4 & 0.14 \\
\hline LiNbO $_{3}$ & 216 & 0.054 & 4 & 3 & 580 & 0.041 & 2 & 1.27 \\
\hline
\end{tabular}

The highest drilling rate was obtained by $\mathrm{LiNbO}_{3}$ sampler where, under a drive voltage of $\sim 580 \mathrm{Vrms}$, the drilling rate was $\sim 1.4 \mathrm{~mm} / \mathrm{min}$ during the first minute and decreased quickly. Electric current surges were found in the following consecutive runs after 2 minutes. Several cracking of the crystal were found after test. It is believed that electric arcs through the cracks in the broken crystals were the source of the current surges. A photograph of the actuator after being exposed to the $500^{\circ} \mathrm{C}$ environment is shown in Figure 8. Post drilling measurements of the electrical properties of the actuator showed that the resistance between the electrodes was greatly reduced even at room temperature.

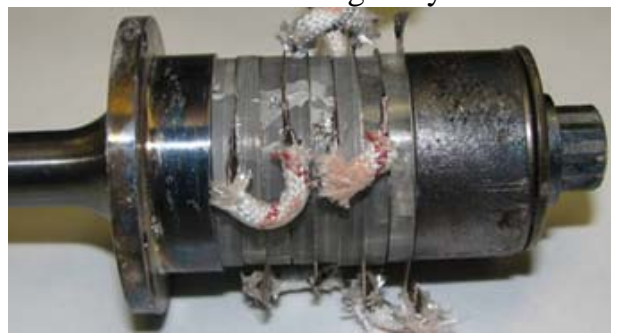

Figure 8: Photograph of the first HT USDC actuator breadboard. The piezoelectric stack was made of $\mathrm{LiNbO}_{3}$ and was tested at $500^{\circ} \mathrm{C}$.

Both samplers that were made of the PZ-46 and the Bismuth Titanate (produced by PSU) actuators worked at $500^{\circ} \mathrm{C}$ and were found to be relatively robust. Only a few cracks in the ceramic rings were found after the tests. However, the 
achieved drilling rates were lower than with the $\mathrm{LiNbO}_{3}$ actuator. Between the two, the Bismuth Titanate actuator showed better performance than the PZ46.

Based on these test results it was decided to make larger diameter samplers using PSU BT and LiNbO3 for improvement of the performance. Several measures intended to avoid cracks in the assemble actuators were applied to the new transducers.

\subsection{Samplers actuated by 1.5” diameter Bismuth Titanate}

After assembling 1.5” diameter bismuth titanate discs (made by PSU) the samplers were tested at room temperature drilling basalt. The bit used was made of tungsten carbide with 6 teeth. The preload for free-mass was equal to WOB of $15 \mathrm{~N}$ and the sampler was driven by $900 \mathrm{Vrms}$ with a duty cycle of 0.5 seconds per second. The current was around $1 \mathrm{~A}$ and power was $\sim 77 \mathrm{~W}$. The penetration rate at the first $0.5 \mathrm{~min}$ was $\sim 0.15 \mathrm{~mm} / \mathrm{min}$, however in a subsequent drilling experiment, it dropped to very low rate. At $5 \mathrm{~min}$, the bit was rotated slowly by hand and a drilling rate was stable at $\sim 0.24 \mathrm{~mm} / \mathrm{min}$. The rotation eliminated the formation of the tooth-print at the bottom of the hole and it helped increasing the drilling efficiency.

Then, a USDC-based sampler with 1.5” diameter bismuth Titanate was tested to drill a brick sample at $460^{\circ} \mathrm{C}$. The bit assembly consisted of two teeth and a center bar extending to outside of the furnace was used and the bit was manually rotated at a low speed around 4-5 rpm. The WOB was $20 \mathrm{~N}$ and the preload for free-mass was $17 \mathrm{~N}$. The sampler was driven in duty cycle of 0.5 seconds per second. The bit was much easier to rotate when the sampler was active than inactive. The torque needed for the rotation was estimated at less than 0.2 inch-lbs. The test results for 26 $\mathrm{mm}$ thick brick sample are shown graphically in Figure 9. A starting hole of about $1 \mathrm{~mm}$ deep was drilled initially at room temperature and the drilling was continued at high temperature. The driving frequency was $20.9-21.0 \mathrm{kHz}$, the averaged voltage $400 \mathrm{Vrms}$, and the averaged current was $0.69 \mathrm{~A}$. The power factor was low $\sim 0.094$ and the averaged power was 26 Watts. The sampler drilled through the sample in 21 active minutes and reached the other end of the brick sample and the average drilling rate was $1.2 \mathrm{~mm} / \mathrm{min}$. The decrease of the drilling rate with time or depth is clearly seen in Figure 9. A room temperature test after the high temperature drilling showed a reasonable rate compared to previous tests. The misalignment, which can result in friction between the bit and hole, might be the major cause of the decrease of the drilling rate.

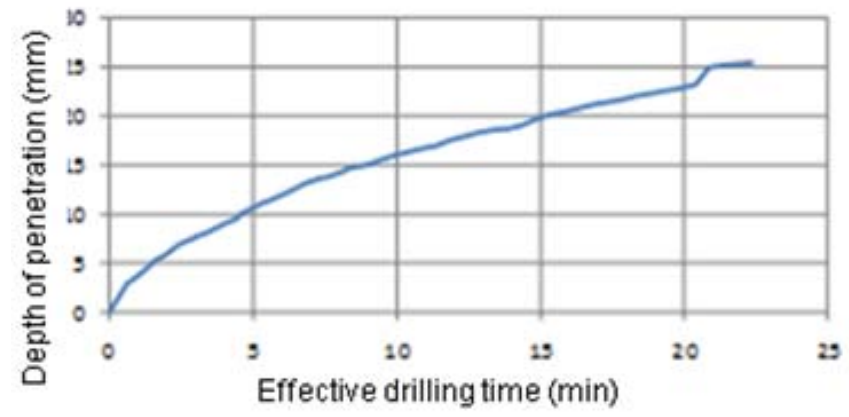

Figure 9: Penetration vs. time for drilling a brick at $460^{\circ} \mathrm{C}$ using a USDC-based sampler with $1.5^{\prime}$ diameter bismuth Titanate.

\subsection{Sampler actuated by 2" diameter $\mathrm{LiNbO}_{3}$}

A sampler that is actuated by 2" diameter $5 \mathrm{~mm}$ thick $\mathrm{LiNbO}_{3}$ was constructed with circumferential preload (estimated to be $30 \mathrm{MPa}$ ). In addition, 2 de-poled PZT rings were added to provide further protection of the LN stack from cracking. A self-rotating bit assembly was used and the bit assembly had an extension bar through a hole at the center of the actuator. A spring mounted between the extension bar and the back of the actuator provided a preload to the freemass. The self-rotating bit worked but stopped frequently at high temperature test. To keep the bit rotating we helped the rotation manually. The tests that were conducted are as follows:

- At room temperature a rate of 7 - $8 \mathrm{~mm} /$ minute was measured in a brick sample.

- The rate for drilling at $460^{\circ} \mathrm{C}$ is shown in Figure 10. The driving frequency, voltage and weight-on-bit were adjusted during the test while seeking for the best operating parameters.

- In the last 5 minutes of the test - the drive frequency was $19.9 \mathrm{kHz}$, the average power was $78 \mathrm{~W}$, the weight-on-bit was $18.7 \mathrm{~N}$, the preload for free-mass was $40 \mathrm{~N}$, and the average drilling rate was $0.43 \mathrm{~mm} / \mathrm{min}$. 
- The test ended due to the fact that the power amplifier shut off. After testing it was noticed that the shape of the impedance curves of the transducer at room temperature were similar but the resonance frequency was lower than the resonance frequency measured before the test. Drilling tests at room temperature showed large degraded performance and cracks were seen on the stack.

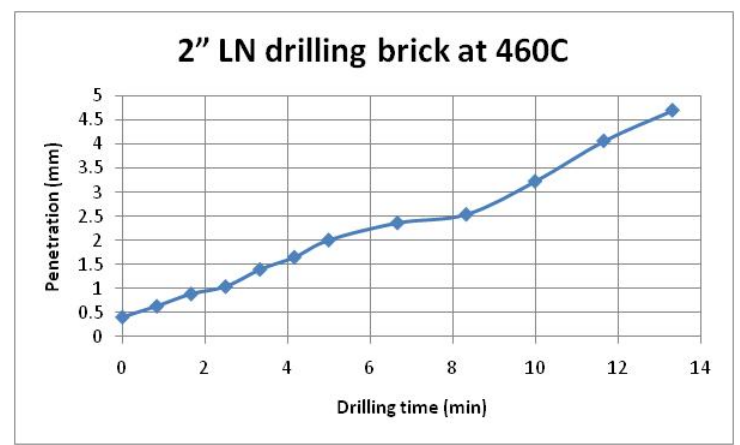

Figure 10: The penetration depth as a function of drilling time for Drill \#2.

\section{Summary and Conclusions}

In this study, HT samplers were developed that can operate at temperatures as high as $500^{\circ} \mathrm{C}$. The major accomplishment has been that using the Bismuth Titanate actuator material (made by PSU) a sampler was developed that drilled brick to a depth of $25 \mathrm{~mm}$ in 21 accumulated minutes (accounting for the use of duty cycling). The sampler is based on the Ultrasonic/Sonic Driller/Corer (USDC) and it was demonstrated that a modified breadboard sampler can be operated at the Venus ambient conditions. The developed drill was designed with our novel method of operating the USDC as a rotary-hammer where the rotation is induced by the vibration of the piezoelectric actuator. The drill was actuated by piezoelectric materials that have higher Curie temperature than the temperature on Venus. Key benefits of the developed sampler is its ability to create fine powdered cuttings that are ideal for XRD/XRF analysis and many other analytical instruments. In this study, the high pressure on Venus was not considered since it is not expected to affect the operation of the USDC.

Six different USDC breadboard were produced including: 1.0 inch diameter LiNbO3, Pz46 (commercially made by formerly Ferroperm and currently owned by Meggitt), Bismuth Titanate made by PSU in a rotary-hammering configuration; 1.5 inch Bismuth Titanate made by PSU; 2.0 inch diameter LiNbO3 and 2.0 inch diameter LiNbO3 in a rotary-hammering configuration. Isothermal tests of LiNbO3 piezoelectric discs at $500^{\circ} \mathrm{C}$ were made and showed high stability with no measureable change in properties after 1000 hours of exposure. Another accomplishment that can be reported is the finding that the Bismuth Titanate with various doping of tungsten shows thickness coupling coefficient of about 15 to $20 \%$ at $500^{\circ} \mathrm{C}$ compared to the room temperature value. To simplify the analysis of the HT USDC actuator and designing effective samplers, equivalent circuit models were developed for the piezoelectric rings as lumped circuit components. To tests the operation of the drill, a HT testbed was developed capable of operating significantly beyond the ambient temperature on Venus, i.e., $460^{\circ} \mathrm{C}$. Brick samples were used in this study since they showed more uniform properties at high temperatures than the natural rocks.

In this task, we had many significant challenging issues to overcome including cracking when using $\mathrm{LiNbO}_{3}$ crystal as an actuator of a USDC-based sampler. Based on the lessons learned, making a sampler using Bismuth Titanate actuator that performs consistently will require improvements to the self-rotating bit mechanism and the power of the actuator will need to be increased to a level that allows it to effectively drill hard rocks.

\section{ACKNOWLEDGEMENT}

Research reported in this manuscript was conducted at the Jet Propulsion Laboratory (JPL), California Institute of Technology, under a contract with the National Aeronautics and Space Administration (NASA). This task was funded by the NASA Planetary Instrument Definition and Development Program (PIDDP).

\section{REFERENCE}

Bao X., J. Scott, Y. Bar-Cohen, S. Sherrit, S. Widholm, M. Badescu, T. Shrout, and B. Jones, "Ultrasonic/sonic drill for high temperature application,” SPIE Sensors and Smart Structures Technologies for Civil, Mechanical, and Aerospace Systems Smart Structures and Materials Symposium, Paper 7647-116, San Diego, CA, March 8-11, 2010. 
Bar-Cohen Y. and K. Zacny (Eds.), "Drilling in Extreme Environments - Penetration and Sampling on Earth and Other Planets,” Wiley - VCH, Hoboken, NJ, ISBN-10: 3527408525, ISBN-13: 9783527408528, (2009) pp. 1-827.

Gershman R., R.A Wallace, “Technology Needs of Future Planetary Missions, Acta Astronautica, 45, Nos. 4-9, pp. 329335, 1999

Honeybee, "High temperature motor specification sheet," downloaded Jan 2011 http://www.honeybeerobotics.com/images/stories/pdf/HTM_Data_Sheet.pdf

Sherrit S., X. Bao, Y. Bar-Cohen, and Z. Chang, "Resonance Analysis of High Temperature Piezoelectric Materials for Actuation and Sensing," SPIE Smart Structures and Materials Symposium, Paper \#5388-34, San Diego, CA, March 15-18, 2004

Troy R., Private communication, Jan 2011 